\title{
The Cepheids Variable Stars Population in the Local Group Dwarf Irregular Galaxy Pegasus
}

\author{
I. Meschin ${ }^{1}$, C. Gallart ${ }^{1}$, S. Cassisi ${ }^{2}$, A. Aparicio ${ }^{1,3}$ and A. Rosenberg ${ }^{1}$ \\ ${ }^{1}$ Instituto de Astrofísica de Canarias. 38200 La Laguna. Tenerife, Canary Islands. Spain \\ email: imeschin@iac.es \\ ${ }^{2}$ INAF - Osservatorio Astronomico di Teramo, Teramo, Italy ${ }^{3}$ Departamento de Astrofísica, \\ Universidad de La Laguna, Tenerife, Spain
}

\begin{abstract}
We present the result of a search for Cepheids in the Local Group dwarf irregular galaxy (DIG) Pegasus. We identified 20 Cepheid stars with periods $0^{d} .6<P<15^{d}$. 3 . We have compared them with previously published Cepheids and we have carried out an accurate completeness analysis in order to obtain the fraction of them which are lost. Using their periodluminosity relation we obtain a distance for Pegasus of $950 \pm 30 \mathrm{Kpc}$.
\end{abstract}

Keywords. local group galaxies: individual (Pegasus,DDO216,UGC12613) — galaxies: dwarf - galaxies: stellar content — stars: variables: Cepheids

\section{Method and Analysis}

The search for Cepheid was made from a ground-based collection of 79 images (55 V and $24 \mathrm{I}$, seeing $\left.<1^{\prime \prime} .35\right)$ compiled throughout several years (1993-2004).

Data reduction was performed in the IRAF environment. The photometric reduction of the frames was performed using the DAOPHOT-ALLSTAR-ALLFRAME suite of programs (Stetson 1987 and 1994) for PSF-fitting photometry in crowded fields. Due to the inhomogeneity of images, matching is more difficult than usual.

We created a "master" image by interpolating the individual frames and applying a median filter (using MONTAGE2). Variable stars searching was performed in this master image. We made a first selection of variable candidates based in the variability index "var" defined as the ratio of the external $\sigma_{\text {ext }}$ to the internal standard error $\sigma_{\text {int }}$ as calculated by DAOMASTER from the individual magnitudes of the $55 \mathrm{~V}$ images. We selected as variable candidates the stars having var $>3$. In all, 319 stars fulfill this criterion.

\section{Results}

We constructed the color magnitude diagram on the basis of our photometry. Only 204 of our candidates are in the instability strip. The light variations of these candidate pulsating variables were searched for periodicities using a phase-dispersion minimization algorithm (Stellingwerf 1978). We visually inspected the light curves that each of the most likely periods would produce for each variable and we found periods for 43 candidates, but only some of them fulfill certain requirements besides the fact of being located next to the instability strip: good period-luminosity relation and correct location in the galaxy. By this way we discarded $54 \%$ of the candidates, and 20 bona fide Cepheid were retained. The V-band light curves for some of these stars are displayed in Figure 1. 

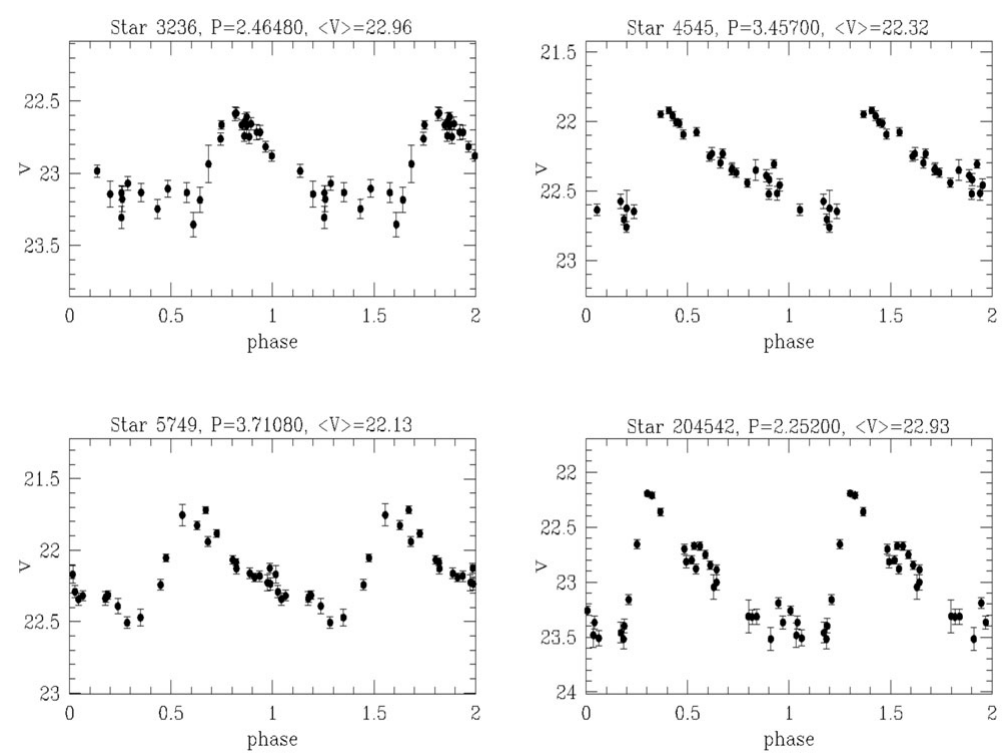

Figure 1. Some of the V-band light curves for the Pegasus Cepheids. Data are repeated over a second cycle for clarity

We carried out an accurate completeness analysis by adding artificial Cepheid stars with the DAOPHOT routine ADDSTAR to the original frames, in order to check the fraction of Cepheids lost. Cepheids magnitudes were simulated using IAC-Star (Aparicio $\&$ Gallart, 2004) and VARFINDER codes, also we used the prototype's $\delta$ Cepheid to introduce the pattern of variability. A completeness factor as a function of light curves amplitude was found. Briefly, we are finding only $64 \%$ of the Cepheids that exists in Pegasus.

Hoessel et al. (1990) provided identification list of 10 Cepheids in Pegasus. However we find in agreeement with Aparicio (1994), that they are badly located in DCM (in the zone of red giants), far away of instability strip. None of them appeared in our search like candidates, in spite of that we checked their light curves without finding any convincing light curves. On the aim of only 2 nights data, Aparicio (1994) suggested 6 candidates to Cepheids. Two of them were also candidates in our search, but we accepted only one as Cepheid.

The distance to Pegasus has been obtained using our 20 Cepheid stars. We derived $950 \pm 30 \mathrm{Kpc}$. This measurement agrees with the earlier work by Aparicio (1994) who obtained from the absolute magnitude of the RGB tip (TRGB) $d=950 \pm 30 \mathrm{Kpc}$ and with the more recent study by McConnachie et al. (2005), $d=919 \pm 30 \mathrm{Kpc}$, also obtained from TRGB.

\section{References}

Aparicio, A. 1994, ApJ 437, L27

Aparicio, A., \& Gallart, C. 2004, AJ 128, 1465

Hoessel J. G., Abbott M. J., Saha A., Mossman A. E. \& Danielson G. E. 1990, AJ 100, 1151

McConnachie, A. W., Irwin, M. J., Ferguson, A. M. N., Ibata, R. A., Lewis, G. F. \& Tanvir, N. 2005, MNRAS 356, 979

Stellingwerf, R. F. 1978, ApJ 224, 953

Stetson, P. B. 1987, PASP 99, 191

Stetson, P. B. 1994, ARAA 106, 250 